\title{
University mathematics students' self-efficacy beliefs about proof and proving
}

\author{
Antti Viholainen ${ }^{1}$, Timo Tossavainen ${ }^{2}$, Hanna Viitala ${ }^{2}$ and Maria Johansson ${ }^{2}$ \\ ${ }^{1}$ University of Eastern Finland, Finland \\ ${ }^{2}$ Luleå University of Technology, Sweden
}

\begin{abstract}
We examine university students' motivation and self-efficacy beliefs about proof and proving, i.e., beliefs about personal abilities to understand, construct and present mathematical proofs, as well as their certainty about self-produced proofs. The sample of the study consists of 29 Finnish and Swedish students who were studying mathematics in tertiary level. The results show that the students were highly motivated to learn to understand and construct proofs, but they were more uncertain about their proving skills. Moreover, the study revealed reasons for the uncertainty about proving achievements. Most of the reasons are caused by deficiencies with respect to knowledge of, understanding about or experience from proof and proving.
\end{abstract}

Keywords: mathematics student teacher, motivation, proof, self-efficacy

\author{
Article Details \\ LUMAT General Issue \\ Vol 7 No 1 (2019), 148-164 \\ Received 14 May 2019 \\ Accepted 16 October 2019 \\ Published 29 October 2019 \\ Pages: 17 \\ References: 26 \\ Correspondence: \\ antti.viholainen@uef.fi
}

https://doi.org/10.31129/ LUMAT.7.1.406

\section{Introduction}

Proving is a mathematical activity that belongs essentially to mathematics, yet it is often considered challenging. In the construction of a proof, creative reasoning and the invention of new ideas are often required instead of building on imitative reasoning or ready-made examples or step-by-step algorithms (Lithner, 2008). Succeeding in this, call for a deep understanding of mathematical structures and quite a lot of monitoring and evaluation both during and after the proving process (Flavell, 1979).

In this study, we will analyse university students' beliefs about their own competencies to understand and construct mathematical proofs. These beliefs constitute self-efficacy beliefs about proof and proving. In general, according to Bandura (1997), self-efficacy consists of beliefs about personal abilities to perform a task and reach the established goals. Self-efficacy influences students' effort, persistence, perseverance and use of strategies (Pajares, 1996). The present study is motivated by the awareness that self-efficacy can be considered to play a significant role also in mathematical proving. Furthermore, the study is motivated by the differences in the attitudes towards a teacher's profession between Nordic countries. The appreciation of teachers is still quite high in the Finnish society but, in Sweden, 
the case is somewhat opposite (OECD, 2013). This may affect student teachers' selfefficacy and view of mathematics. Our aim is to examine whether there are such differences between Finnish and Swedish mathematics student teachers in the context of proving.

\section{Role of proof in mathematics education}

From the pedagogical perspective, the most important reason why proof and proving are included in the learning goals of mathematics is probably the explanationfunction (deVilliers, 1990; Hanna, 2000). According to de Villiers, the explanationfunction means that a proof provides an insight why something is true. It explains connections between mathematical structures, and, hence, it supports deep conceptual understanding. Viholainen, Lepik, Hemmi, Asikainen, and Hirvonen (2018) found that also university students in Estonia and Finland consider support for understanding the most important reasons to study proof and proving. Other reasons they frequently mentioned are the development of thinking, reasoning and argumentation skills. This refers to transfer-function of proof, which means that via proofs it is possible to learn skills that are useful also in another context (Hemmi, 2006).

The importance of proof increases considerably when a student moves from the upper secondary school to studying mathematics in university. This, together with the more formal nature of mathematics, may cause cognitive conflicts and culture shocks for a student (Clark \& Lovric, 2009; Tall, 1995). It is not surprising that university students often have difficulties with proof and proving (Hemmi, 2006; Reid \& Knipping, 2010; Tossavainen \& Luostarinen, 2004). However, Viholainen et al. (2018) have showed that students at tertiary level appreciate the importance of proof both in school mathematics and in mathematics in general. Students consider that the support that proving gives them in understanding mathematics is the most important reason to study proof and proving. On the other hand, the level of proving skills and view of mathematics may be dependent: Tossavainen and Luostarinen (2004) noticed that students who were not yet skilful in proving viewed mathematics as a static system, whereas students who were more skilful considered the nature of mathematics more dynamic.

There are quite large differences between countries with respect to the role of proof in school mathematics. Hemmi, Lepik and Viholainen (2013) found from 
Estonian, Finnish and Swedish curricula documents for grades 1-12 that, in the Swedish curriculum, proof and proving were clearly less emphasized than in the Estonian and Finnish ones. In the Finnish curriculum, several proof-related elements were included without mentioning explicitly the concepts of proof or proving. However, especially in Finland, the role of proof has varied quite much through time (Mäkelä, 2011). Bergwall and Hemmi (2017) analyzed Finnish and Swedish textbooks used in integral calculus at upper secondary level with respect to proof-related reasoning. Their study revealed that proofs were more visible in the Finnish textbooks than in the Swedish ones. Expository sections in the Finnish textbooks offered more opportunities to learn proof and proving, but the Swedish textbooks included hardly any general proofs. In both countries, the proportion of proof-related exercises in textbooks was low (about $10 \%$ ), and the number of tasks that explicitly ask students to provide a proof were minimal. However, the exercises in the Swedish textbooks reflected a higher variation of proof-related reasoning.

\section{Self-efficacy beliefs about proof and proving}

Self-efficacy and student activities affect one another. First, self-efficacy can influence effort and persistence by affecting the choice of activities (cf. Pajares, 1996). This effect can be positive, but it can also hinder learning. Second, self-efficacy beliefs develop through interpretations of prior experiences. These mastery experiences are the most powerful source for self-efficacy (Bandura, 1997; Usher \& Pajares, 2009). They are formed over a longer time period, which makes self-efficacy beliefs resistant to change (Bandura, 1997; cf. trait aspect in Hannula, 2011). Vicarious experiences, together with peer feedback, influence self-efficacy through social situations. Additionally, physiological and emotional arousals such as anxiety or the feeling of flow has an influence on self-efficacy (Bandura, 1997; Usher \& Pajares, 2009; Hannula, 2011).

Self-efficacy beliefs are context specific (Bandura, 1997) and often studied as quite stable trait beliefs. For example, the reciprocal connection between self-efficacy and mathematics performance is well established in many contexts (e.g. Lee, 2009; Williams \& Williams, 2010). In addition to academic achievement in mathematics, self-efficacy has been shown to have close connections also with motivation and selfconfidence (see e.g. Zimmerman, 2000). The strength of perceived self-efficacy to 
understand or perform a task is often studied through students' self-confidence (cf. Bandura, 1997).

In proving, there are three different central activities: reading a given argument, constructing a novel argument, and presenting an available argument (Mejía-Ramos \& Inglis, 2009). Different mathematical competencies are needed in these activities. Reading a given argument requires that a learner can read mathematical text, understand the content of the argument, and that a learner is capable to evaluate if the argument is correct and sufficient. Construction of a novel argument involves deep understanding of the content, and it often demands the use of mathematical thinking and problem-solving skills. Presenting an available argument presumes competence to present an argument in an understandable and acceptable way.

By applying Bandura's (1997) definition of self-efficacy to the activities of proving presented by Mejía-Ramos \& Inglis (2009), we interpret that self-efficacy in the context of proof and proving consists of the following elements:

- Beliefs about personal abilities to understand mathematical proofs and evaluate them critically.

- Beliefs about personal abilities to construct mathematical arguments and combine them to a proof.

- Beliefs about personal abilities to present a mathematical proof in an acceptable way.

\section{Research questions and method}

The subjects of this study are university students in mathematics from one Finnish and one Swedish university. The aim is to get answers to the following questions:

1. How motivated are students in mathematics to study skills needed for proving?

2. How do students perceive their abilities in tertiary mathematics in general and, especially, their abilities to understand and produce mathematical proofs?

3. If students manage to produce mathematical proofs, how convinced are they about the correctness of their solutions?

4. If the students are unsure about the correctness of their self-produced proofs, what kind of reasons do they see for this uncertainty? 
The first question deals with students' motivation to study proof and proving. Because motivation is an important prerequisite for learning, this question offers background for the other questions. The second question is explicitly about the students' self-efficacy beliefs about proof and proving (cf. Section 3). In this study, only the abilities to understand and produce mathematical proofs are studied as a separate question, because we assume that the production of a proof includes both the construction and presenting of the proof. The third question addresses to students' self-efficacy and self-assessment of their own performance. The last question aims at revealing students' conceptions about reasons for potential uncertainty with respect to their self-produced proofs.

The participants in this study have already some experiences from studying university mathematics and hence also from proving. The sample consists of 21 Finnish and 8 Swedish third-year students from one Finnish and one Swedish university. Most of the students are student teachers in mathematics (18 students in the Finnish sample and everyone in the Swedish sample). Although the number of participants is small, the sample can be considered quite representative. The Swedish participants make up the whole third year class at the Swedish university, and Finnish participants about $90 \%$ of the same class at the Finnish university. In both countries, the survey was conducted during an ordinary lecture of a compulsory course for student teachers. All the students who were present responded to it on the voluntary basis.

Data were collected with a questionnaire containing both Likert-scale statements and open questions. In the Likert-scale items, a five-point response scale (1= strongly disagree, $2=$ disagree, $3=$ neutral view, $4=$ agree, $5=$ strongly agree) was used. Data for the research questions 1-3 consist of the responses to the Likert-scale statements. Statements were originally presented in Finnish (for Finnish students) and in Swedish (for Swedish students) but are translated into English for this report.

The quantitative analyses of our data included computing standard descriptive measures and effect sizes and applying Student's t-tests. Further, we have calculated Spearman correlation measures and given the percentual distributions of response frequencies. Since the sample size is small, sometimes it is more useful to evaluate differences between the groups with the aid of the effect sizes (Cohen's d) than using t-tests. In certain analyses, we have done so. 
Data for the question 4 comes from the open question where students were asked to analyse possible reasons for their eventual uncertainty about the correctness of a self-produced proof. The responses were analysed by applying data-based content analysis in which the response categories were created based on the data. Also, in this part, the Finnish students answered in Finnish, and the Swedish students in Swedish. In the next section, two researchers have checked the translations of the quotations from Finnish and Swedish responses into English.

\section{Results}

\subsection{Students' motivation for proving}

The results presented in Table 1 indicate that the participating students experienced high motivation for learning activities concerning proofs. Students expressed willingness to learn both to understand and to construct mathematical proofs. The Swedish students indicated a little higher motivation to learn to construct proofs than to understand them. Because of the sample size, this difference is however not statistically significant. In Statement 2, Cohen's d for the means of Finnish and Swedish sample is -0.80, which indicates a large effect size (Cohen, 1988).

Table 1. Students' motivation to learn proving. Means and standard deviations for Statements 1 and 2, and effect sizes (Cohen's d) for the differences between Finnish and Swedish samples.

\begin{tabular}{lccccccc}
\hline \multirow{2}{*}{ Statement } & \multicolumn{2}{c}{ Finland $(\mathbf{n}=\mathbf{2 1})$} & \multicolumn{2}{c}{ Sweden $(\mathbf{n}=\mathbf{8})$} & \multicolumn{2}{c}{ Total $(\mathbf{n}=\mathbf{2 9})$} & $\mathbf{d}$ \\
& Mean & Std. dev. & Mean & Std. dev. & Mean & Std. dev & \\
\hline $\begin{array}{l}\text { S1: I want to learn to understand } \\
\text { mathematical proofs. }\end{array}$ & 4.19 & .87 & 4.38 & .74 & 4.24 & .83 & -.24 \\
$\begin{array}{l}\text { S2: I want to learn to construct } \\
\text { mathematical proofs. }\end{array}$ & 4.24 & .77 & 4.75 & .46 & 4.38 & .73 & -.80 \\
\hline
\end{tabular}

The more detailed percentual distributions of the response frequencies are presented in Table 2. This table shows that none of the respondents were totally unmotivated to study activities concerning proofs. In S1, altogether $83 \%$ of the students responded that their motivation to learn to understand mathematical proofs is at least on the level 4. In S2, all the students evaluated their motivation at least to level 3 , and more than half of them ( $52 \%$ ) evaluated their motivation toward proofproduction to be at the highest level in the five-point scale. 
Table 2. Percentual frequencies of Statements 1 and $2(n=29)$.

\begin{tabular}{cccccccc}
\hline & $\mathbf{1}$ & $\mathbf{2}$ & $\mathbf{3}$ & $\mathbf{4}$ & $\mathbf{5}$ & Mode & Median \\
\hline S1 & 0 & 3 & 14 & 38 & 45 & 5 & 4 \\
S2 & 0 & 0 & 14 & 35 & 52 & 5 & 5 \\
\hline
\end{tabular}

\subsection{Students' conceptions about their general mathematical abilities and abilities concerning proofs}

In Statement 3, the students were asked to evaluate their general mathematical skills. Statement 4 deals with abilities to understand mathematical proofs, and Statement 5 abilities to construct proofs. The results are presented in Table 3.

Table 3. Students' conceptions about their general mathematical abilities and abilities to understand and construct mathematical proofs.

\begin{tabular}{lccccccc}
\hline \multirow{2}{*}{ Statement } & \multicolumn{2}{c}{ Finland $(\mathbf{n = 2 1})$} & \multicolumn{2}{c}{ Sweden $(\mathbf{n}=\mathbf{8})$} & \multicolumn{2}{c}{ Total $(\mathbf{n}=\mathbf{2 9})$} & d \\
& Mean & Std. dev. & Mean & Std. dev. & Mean & Std. dev. \\
\hline $\begin{array}{l}\text { S3: I am skillful in university } \\
\text { mathematics. }\end{array}$ & 3.14 & .91 & 3.13 & .84 & 3.14 & .88 & .01 \\
$\begin{array}{l}\text { S4: I am skillful in understanding } \\
\text { mathematical proofs. }\end{array}$ & 3.10 & .94 & 3.38 & .74 & 3.17 & .89 & -.33 \\
$\begin{array}{l}\text { S5: I am skillful in constructing } \\
\text { mathematical proofs. }\end{array}$ & 2.38 & .74 & 2.25 & .89 & 2.34 & .77 & .16 \\
\hline
\end{tabular}

The results in Table 3 show that students typically assign a score for their general mathematical skills from the middle of the scale. Similarly, they evaluated their abilities to understand mathematical proofs to be at the same level. However, they consider their abilities to construct mathematical proofs remarkably lower than the previously mentioned skills (S3 vs. S5: $\mathrm{t}(28)=-6.33, \mathrm{p}<.001$; $\mathrm{S}_{4}$ vs. S5: $\mathrm{t}(28)=-6.27$, $\mathrm{p}<.001)$. In Statement $\mathrm{S} 4$, the effect size between Finnish and Swedish samples is between small and medium (Cohen, 1988).

Table 4. Percentual frequencies of Statements 3,4 , and $5(n=29)$.

\begin{tabular}{cccccccc}
\hline & $\mathbf{1}$ & $\mathbf{2}$ & $\mathbf{3}$ & $\mathbf{4}$ & $\mathbf{5}$ & Mode & Median \\
\hline S3 & 3 & 17 & 45 & 31 & 3 & 3 & 3 \\
S4 & 0 & 28 & 31 & 38 & 3 & 4 & 3 \\
S5 & 10 & 52 & 31 & 7 & 0 & 2 & 2 \\
\hline
\end{tabular}


The percentual frequencies presented in Table 4 strengthen the already made conclusions that students evaluate their knowledge about university mathematics and proofs quite modestly and their skills in proving being even poorer. The more interesting observation is that the frequencies for the extreme values are again low.

\subsection{How convinced the students are about their proving performances?}

Statement 6 deals with the question how convinced students are about their performance in proving tasks and, in Statement 7, students were asked to compare their experiences of uncertainty between proving tasks and other mathematical exercises. The means and standard deviations of their responses are presented in Table 5. They show that the Swedish students are more critical toward Statement 6 than the Finnish students. Effect size (Cohen's d) is almost medium size (Cohen, 1988). However, in Statement 7, there is no significant difference between the groups. Due to the large variation, the mean difference between Statements 6 and 7 is not statistically significant $(\mathrm{t}(28)=-1.42, \mathrm{p}>.05)$.

Table 5. Students' experience of uncertainty in proving tasks.

\begin{tabular}{|c|c|c|c|c|c|c|c|}
\hline \multirow{2}{*}{ Statement } & \multicolumn{2}{|c|}{ Finland $(n=21)$} & \multicolumn{2}{|c|}{ Sweden $(n=8)$} & \multicolumn{2}{|c|}{ Total $(n=29)$} & \multirow[t]{2}{*}{ d } \\
\hline & Mean & St. dev. & Mean & St. dev & Mean & St. dev & \\
\hline $\begin{array}{l}\text { S6: If I manage to construct a } \\
\text { mathematical proof, I am convinced } \\
\text { about its correctness. }\end{array}$ & 3.24 & .89 & 2.75 & 1.17 & 3.10 & .98 & .47 \\
\hline $\begin{array}{l}\text { S7: In proving tasks, I feel more } \\
\text { uncertainty about the correctness of } \\
\text { my solution than in other types of } \\
\text { exercises. }\end{array}$ & 3.62 & 1.24 & 3.63 & 1.41 & 3.62 & 1.27 & -.01 \\
\hline
\end{tabular}

The more detailed distributions of response frequencies are presented in Table 6. In S6, there was quite a much dispersion in the responses; altogether $28 \%$ of the students responded ' 1 ' or ' 2 ', which indicates that they were not convinced about the correctness of their proving performances. Instead, $35 \%$ responded at least ' 4 ' to this question, which means that they were more often convinced than unconvinced. About two thirds of the respondents (66\%) agreed at least at the level 4 with Statement 7 , showing that they feel more uncertainty in the case of proving tasks than in the case of other mathematical exercises. However, there was a minority who did not feel in this way: Almost one fourth (24\%) responded ' 1 ' or ' 2 ' to this claim. 
Table 6. Percentual frequencies of Statements 6 and $7(n=29)$.

\begin{tabular}{cccccccc}
\hline & $\mathbf{1}$ & $\mathbf{2}$ & $\mathbf{3}$ & $\mathbf{4}$ & $\mathbf{5}$ & Mode & Median \\
\hline S6 & 3 & 24 & 38 & 28 & 7 & 3 & 3 \\
S7 & 7 & 17 & 10 & 38 & 28 & 4 & 4 \\
\hline
\end{tabular}

\subsection{Correlations between the statements}

Covariance between the variables in Statements 1-7 were also studied with the aid of Spearman's correlation coefficient. All pairs for which the correlation is significant, at least, at the level .05 are listed in Table 7.

Table 7. Correlations (Spearman's rho) between the variables S1-S7 ( $n=29)$.

\begin{tabular}{c|c|c}
\hline & Spearman's rho & Sig. \\
\hline S3 - S6 & .53 & $<.01$ \\
S4-S6 & .37 & $<.05$ \\
S5-S6 & .59 & $<.001$ \\
S3-S7 & -.50 & $<.01$ \\
S4-S7 & -.43 & $<.05$ \\
\hline
\end{tabular}

Interestingly, $\mathrm{S} 1$ and $\mathrm{S} 2$, which measure motivation to study proving activities, do not correlate at significance level .05 with the other variables. Variable S6 correlates positively with all the variables concerning abilities in $\mathrm{S}_{3}, \mathrm{~S}_{4}$, and $\mathrm{S}_{5}$. This means, for example, that if a student tends to be convinced about the correctness of his or her proof, he or she probably feels to be skillful in university-level mathematics in general and both in understanding and constructing mathematical proofs. Variable S7 correlates negatively with $\mathrm{S}_{3}$ and $\mathrm{S} 4$. This indicates, for example, that the more skillful a student experiences to be in university mathematics and, especially, understanding mathematical proofs, the less there is difference in his or her uncertainty about the correctness of his or her solutions between proving tasks and other mathematical tasks.

\subsection{Reasons for uncertainty about proof construction}

The open question was formulated as follows: If you experience uncertainty about the correctness of a proof you have constructed, what are the reasons for this? Results presented in Table 8 reveal how many students mentioned each reason for uncertainty in their responses for the open question. Each response may include 
several reasons. Below Table 8, we describe what kind of responses were placed into each response category.

Table 8. Students' reasons for uncertainty about proofs they have constructed $(n=29)$.

\begin{tabular}{l|c}
\hline Reason category & Frequency (\%) \\
\hline Regulating the proving process & 41 \\
Fear of mistakes or deficiencies & 41 \\
Nature of the proof & 24 \\
Understanding of the mathematical content & 28 \\
Presentation & 7 \\
Unspecified reasons & 10 \\
\hline
\end{tabular}

\subsubsection{Regulating the proving process}

In total, $41 \%$ of the respondents somehow mentioned regulating the proving process as a reason for potential uncertainty. The students expressed difficulties either in beginning or in organising the proving process:

"Usually, I am not sure how I should start a proof."

"The biggest problem is that often I do not know how to begin a proof."

"In the case of proofs with antithesis, I experience the proving process as troublesome. It is difficult to see where the process should result in, in order to achieve the desired result."

"Most often, it is difficult to know in advance which areas should be investigated in order to prove an affair."

Students expressed also annoyance for that there rarely exists a ready-made algorithm or a model to be imitated. This makes the proving process difficult:

"I consider proofs as the most difficult things in mathematics, because there exists no specific formula that should be used in specific proofs."

"In many other types of mathematical assignments, there is often something concrete (for example, a formula) which may be applied to solve the problem. Proving is more abstract for me, because here you have to help yourself to find a rule that leads to a proof."

A couple of students referred only to a lack of experience from proving:

"Lack of practicing." 
"Unfamiliarity in producing proofs."

\subsubsection{Fear of mistakes or deficiencies}

Again, $41 \%$ of the student referred to a doubt that a self-produced proof includes some mistakes or deficiencies. These responses revealed that students worry about doing something wrong or they have feelings that the proofs they have produced are insufficient in some way:

"Does my reasoning include errors? Have I unnoticedly assumed something extra?"

"Typing error"

One student speculated about the possibility of making circular reasoning:

"Sometimes I wonder if the whole proof is mere circular reasoning."

Some students referred to difficulties to evaluate whether or not they have used acceptable methods or followed rules in an acceptable way:

"While proving, I often think if it is allowed to use some specific method in that situation."

"Mainly, I hesitate if I have used the correct tools or if I have understood the beginning of the problem correctly / started to solve the problem in a correct way."

"I am not sure about its completeness, or about following of rules."

Due to "... if I have understood the beginning of the problem correctly..." in the second quotation above, this response has been assigned to the class "Understanding of mathematical content”, as well.

\subsubsection{Nature of the proof}

Altogether $24 \%$ of the respondents wrote about difficulties in knowing or understanding the nature of the proof. Some of the students were unsure about the criteria of an acceptable proof:

"Also, you do not always know which things a proof must include." 
"Not knowing of how extensive a proof should be."

"What is enough for a proof?"

One student thought that she was not competent enough to evaluate when a proof is acceptable. This leads to a feeling of uncertainty. The following response was classified both to the class "Nature of proof" and "Uncertainty about correctness".

"I have not yet internalized properly the producing of mathematical proofs, so I cannot evaluate if my proof is firm or if I have thought the issue somehow incorrectly."

Furthermore, the following response was assigned to both categories "Regulating of the proving process" and "Nature of a proof". It illustrates that deficiencies in understanding the nature of proof probably cause problems in organising the proving process as well.

"I do not understand how proving proceeds; and I do not understand how something is proven correctly, if it is explained using difficult terms."

\subsubsection{Understanding of the mathematical content}

Table 8 also shows that $28 \%$ of the students mentioned difficulties in understanding the mathematical content. Many of them considered inadequate understanding of definitions, theorems and lemmas:

"Uncertainty is also caused by not understanding completely all the lemmas and other auxiliary theorems."

"Have I understood the theory correctly, and can I apply it?"

"Probably I have understood some definition wrongly, or I am unsure if I have followed rules.”

Some students reflected uncertainty in understanding the assignment. In the case of proving tasks, it is reasonable to include these to uncertainties in understanding the content:

"You do not understand the question completely."

"That I have not understood the problem." 


\subsubsection{Presentation}

Two students ( $7 \%$ ) raised doubts about the presentation of a proof:

"About clarity."

"Formulation of a proof."

\subsubsection{Unspecified reasons}

Three responses (10 \%) included statements that could not be classified into the above-mentioned categories. These responses were somewhat vague, and thus it was not possible to interpret them reliably enough.

One student wrote that her proofs proves actually nothing for her. However, she did not specify if she had problems, for example, either in understanding the mathematical content or the structure of a proof:

"The proof that I have constructed proves nothing to me."

Two other students referred to deficiencies in their knowledge without specifying that any more:

"Deficient knowledge."

"My knowledge is not good enough in order to have an understanding whether I have done right or wrong."

The latter response includes a reference also to uncertainty about the correctness, and, therefore, it has been classified also to the class "Uncertainty about correctness". However, the unspecified reference to lack of knowledge has been set to the class "Unspecified reasons".

\section{Discussion}

The results of this study show that students are highly motivated to learn to understand and to construct proofs. Almost none of them expressed low motivation to learn these skills. Compared to that, the participating students expressed more uncertainty when they evaluated their competencies to understand and produce mathematical proofs. Especially, they seemed to be doubtful about their skills to 
construct mathematical proofs. In line with this, the students were not very convinced about the proofs they possibly have managed to construct. However, the variation in this issue was quite large, and it seemed that the Swedish students were more uncertain about their proof constructions than their Finnish peers. In our data, certainty about self-produced proofs correlates both with estimates of mathematical competencies in general and with estimates of proving competencies.

According to our findings, most often the reasons for uncertainty about selfproduced proofs are problems in regulating the proving process or a fear of making mistakes. Several students told about their difficulties in understanding the mathematical content or the nature of proof. Nonetheless, only a few students explicitly mentioned difficulties with the presentation of proofs.

Earlier studies (Hemmi, 2006; Reid \& Knipping, 2010; Tossavainen \& Luostarinen, 2004) have reported, on one hand, on students' challenges with respect to proof and proving in tertiary mathematics, and, on the other hand, about university students' high appreciation toward proof and proving (Viholainen, Lepik, Hemmi, Asikainen \& Hirvonen, 2018). Challenges were met also in this study: The results of the survey show that most of the students considered proof and proving quite a challenging area in mathematics. This was reported also in the responses for the open question. Despite of these challenges, the students expressed high motivation to study proof and proving. This motivation may indicate high appreciation towards proof and proving. Another plausible explanation for high motivation to learn proving may be the fact that this skill is needed, for example, in the exams of mathematics courses in university. Unfortunately, our data does not allow us to study to which extent the students' motivation toward proving skills is internal and to which extent external motivation.

Based on the results of this study, mathematics students' self-efficacy beliefs about their competence to understand proofs is, on the average, at the medium level and beliefs about their competence to construct proofs a little lower than that. The analysis of the responses to the open question motivates us to conclude that this seems to be especially due to low self-efficacy in the context of constructing proofs. Most of the explicitly mentioned reasons are based on students' earlier experiences from proving. Typically, they are caused by inadequate knowledge or insufficient understanding, and they are mainly cognitive by nature. These are factors that can be affected through teaching. Our findings give some clues about how the development of students' selfefficacy with respect to proving competencies could be supported. For example, the 
findings related to understanding the nature of proof or difficulties to know when a proof is correct, imply a need to discuss about proof more explicitly in teaching. Hemmi (2008) has pointed out that, especially in Sweden, explicit discussion about proof and proving is often avoided during the lectures, yet different aspects of proof may come out indirectly in calculations etc. According to Hemmi, the lack of explicit discussion about proof may lead to a situation where students are unaware or uncertain about the nature of proof.

We encourage teachers to invest more on an explicit debate about proofs, since from the perspective of our discussion, it should help students to learn how to regulate the proving process. There is evidence (e.g. Attorps, Björk, Radic \& Tossavainen, 2013) that, also at tertiary mathematics education, even small variation in teaching approaches and in the way of explaining the goals of teaching may already have a significant impact on that how students' argument for their reasoning and, thus also on students' self-efficacy.

Further, rehearsing proving in practice is also very recommendable. For example, a recent study from the first-year engineering students in Sweden (Tossavainen, Rensaa, and Johansson, 2019) shows that students whose view of mathematics is strongly based on exact reasoning perform significantly better than those whose view of mathematics focuses more on applications. Their data indicate a positive relationship between the high appreciation of proving and self-efficacy, too. As well, our findings above show that students' uncertainty with proofs are more due to a lack of experience than a lack of interest or a disability to see the value of proving.

\section{References}

Attorps, I., Björk, K., Radic, M., \& Tossavainen, T. (2013). Varied ways to teach the definite integral concept. International Electronic Journal of Mathematics Educations, 8(2-3), 8199.

Bandura, A. (1997). Self-efficacy: The exercise of control. New York, NY: W. H. Freeman.

Bergwall, A., \& Hemmi, K. (2017). The state of proof in Finnish and Swedish mathematics textbooks - Capturing differences in approaches to upper secondary integral calculus. Mathematical Thinking and Learning 19(1), 1-18. DOI: 10.1080/10986065.2017.1258615

Clark, M., \& Lovric, M. (2009). Understanding secondary-tertiary transition in mathematics. International Journal of Mathematical Education in Science and Technology, 40(6), 755776. DOI: 10.1080/00207390902912878

Cohen, J. (1988). Statistical power analysis for the behavioral sciences. Mahwah, NJ: Lawrence Erlbaum.

deVilliers, M. (1990). The role and function of proof in mathematics. Pythagoras, 23, 17-24. 
Flavell, J. H. (1979). Metacognition and cognitive monitoring. A new area of cognitivedevelopmental inquiry. American Psychologist, 34, 906-911. DOI: 10.1037/0003066X.34.10.906

Hanna, G. (2000). Proof, explanation and exploration: an overview. Educational Studies in Mathematics, 44, 5-23.

Hannula, M. S. (2011). The structure and dynamics of affect in mathematical thinking and learning. In M. Pytlak, T. Rowland, \& E. Swoboda (Eds.), Proceedings of the Seventh Congress of the European Society for Research in Mathematics Education (pp. 34-6o). Rzeszów, Poland: University of Rzeszów.

Hemmi, K. (2006). Approaching proof in a community of mathematical practice. Doctoral thesis. Stockholm, Sweden: Stockholm University. Retrieved from http://www.divaportal.org/smash/get/diva2:189608/FULLTEXTo1.pdf

Hemmi, K. (2008). Students' encounter with proof - the condition of transparency. ZDM - The International Journal on Mathematics Education. The Special Issue on Proof, 4O, 413-426.

Hemmi, K., Lepik, M., \& Viholainen, A. (2013). Analysing proof-related competences in Estonian, Finnish and Swedish mathematics curricula -towards a framework of developmental proof. Journal of Curriculum Studies, 45(3), 354-378.

Lee, J. (2009). Universals and specifics of math self-concept, math self-efficacy, and math anxiety across 41 PISA 2003 participating countries. Learning and Individual Differences, 19, 355365. DOI: 10.1016/j.lindif.2008.10.009

Lithner, J. (2008). Research framework for creative and imitative reasoning. Educational Studies in Mathematics, 63(3), 255-276. DOI: 10.1007/slo649-007-9

Mejía-Ramos, J. P., \& Inglis, M. (2009). Argumentative and proving activities in mathematics education research. In F.-L. Lin, F.-J. Hsieh, G. Hanna \& M. de Villiers (Eds.), Proceedings of the ICMI-study 19 conference: Proof and proving in mathematics education, (Vol. 2, pp. 8893). Taipei, Taiwan: The Department of Mathematics, National Taiwan Normal University.

Mäkelä, V. (2011). Todistaminen suomalaisessa ja etelä-korealaisessa koulumatematiikassa. [Proving in Finnish and South Korean school mathematics]. Master's thesis. Tampere, Finland: University of Tampere, Department of Mathematics and Statistics.

OECD. (2013). TALIS Country Notes, Country Profiles and National Reports. Retrieved from http://www.oecd.org/education/school/talis-country-notes-and-country-profiles.htm

Pajares, F. (1996). Self-efficacy beliefs in academic settings. Review of Educational Research, 66(4), 543-578. DOI: 10.3102/00346543066004543

Reid, D.A., \& Knipping, C. (2010). Proof in mathematics education: research, learning and teaching. Rotterdam, Holland: Sense Publishers.

Tall, D. (1995). Cognitive growth in elementary and advanced mathematical thinking. In D. Carraher \& L. Miera (Eds.), Proceedings of X1X International Conference for the Psychology of Mathematics Education, (Vol. 1, pp. 61-75). Recife, Brazil: Universidade Federal de Pernambuco.

Tossavainen, T., \& Luostarinen, K. (2004). Peruskoulun matematiikanopettajaksi opiskelevien todistamistaidot ja matematiikkakuva. In K. Merenluoto \& M. Mikkilä-Erdmann (Eds.), Learning research challenges the domain specific approaches in teaching: A symposium for research on teaching and learning Turku 14.5.2004, (pp. 88-99). Turku, Finland: University of Turku, Department of Teacher Education.

Tossavainen, T., Rensaa, R. J., \& Johansson, M. (2019). Swedish first-year engineering students' view of mathematics, self-efficacy and motivation and their effect on task performance. International Journal of Mathematical Education in Science and Technology. DOI: 10.1080/0020739X.2019.1656827 
Usher, E. L., \& Pajares, F. (2009). Sources of self-efficacy in mathematics: A validation study. Contemporary Educational Psychology, 34(1), 89-101. DOI: 10.1016/j.cedpsych.2008.09.002

Viholainen, A., Lepik, M., Hemmi, K., Asikainen, M., \& Hirvonen, P. (2018). Research study about Estonian and Finnish mathematics students' views about proof. In E. Norén, H. Palmér, H., \& A. Cooke (Eds.), Nordic Research in Mathematics Education, Papers of NORMA 17, (pp. 119127). Göteborg, Sweden: Swedish Society for Research in Mathematics Education.

Williams, T., \& Williams, K. (2010). Self-efficacy and performance in mathematics: Reciprocal determinism in 33 nations. Journal of Educational Psychology, 102(2), 453-466. DOI: 10.1037/a0017271

Zimmerman, B. J. (2000). Self-efficacy: An essential motive to learn. Contemporary Educational Psychology, 25(1), 82-91. DOI: 10.1006/ceps.1999.1016 